\title{
Estrategias y políticas para un desarrollo sostenible
}

\author{
Maritza Margheritt|* \\ Marinela Colina**
}

\section{Resumen}

De la necesidad que se tiene de lograr un desarrollo económico teniendo en cuenta el daño que se le puede hacer al ambiente, se han elaborado algunos modelos que permiten desarrollar estrategias, politicas y tomar decisiones para la utilización eficiente de los recursos naturales que faciliten el camino hacia un desarrollo sostenible. En nuestros palses se presenta la necesidad de seguir un modelo de desarrollo que no implique la disminución del stock de capital natural, en vista de la importancia ecológica que tienen nuestros paises a nivel mundial y que representan el mantenimiento de la vida en el planeta. En el presente trabajo se hace una revisión de estos modelos y la aplicación de los mismos, y se plantean algunas alternativas para desarrollar estrategias y políticas que permitan el mantenimiento del medio ambiente sin descartar el desarrollo.

Palabras claves: Desarrollo sostenible, Stock de capital natural, Estrategias, Políticas.

\section{Strategies and policies for a sustainable development}

\section{Abstract}

Considering the need to plan developing economies knowing the impact on the environment, some models have been designed so as to develop strategies, policies and

Recibido: 21-03-96 . Aceptado: 31-05-96

- Economista. Universidad del Zulia. Master en Economfa de la Energia y del Ambiente E.N.I. Milán-Italia.

** Profesor Titular. Laboratorlo de Química Ambiental. Facultad Experimental de Ciencias. Universidad del Zulla. Maracaibo 4011. Zulla.

Autor para la correspondencia. 
decision making to handle the natural resources, making a common front between countries looking for a profit without destroying the environment. Our countries need to follow a development model which does not involve a depletion of the natural capital stock, given the importance of the South American ecologies which represent the life susteinance on the planet. In this work, we made a review of the concepts needed to follow a development model that does not imply the destruction of our environment. We also recomended development strategies and policies that can mantain the environment in harmony with the development.

Key words: sustainable development, natural capital stock, strategies, policies.

\section{Introducción}

La necesidad que tenemos por conocer más cada día acerca del medio ambiente, en un mundo en constante "desarrollo" nos ha llevado a tratar de sintetizar algunas de las ideas más importantes en relación al problema ambiental, desde el punto de vista económico.

Actualmente los paises exploran el concepto de desarrollo sustentable, con la idea de continuar mejorando la calidad de vida con menor consumo de los recursos. Sabemos de antemano que el ambiente para los economistas es un conjunto de recursos económicos, sin embargo, existe un limite a la posibilidad de utilizar estos recursos provenientes de la tierra. Para comenzar a entender este nuevo juego que ha creado el hombre se plantean las siguientes preguntas:

1.- Hasta qué punto están dispuestos a pagar los agentes contaminantes (individuo, empresa, Estado) para evitar los daños al ambiente?

2.- Hasta que punto se está dispuesto a pagar para disminuir una unidad de contaminación?

3.- En el caso de ser el agente contaminado, Cuánto se desea como re- compensa? Cuánto sería el mínimo dispuesto a aceptar para cubrir este daño?

Se sabe lo diffcil que resulta compaginar un sistema económico con el medio ambiente de manera que ambos $\cos \theta$ xistan en equilibrio. Hasta ahora esto no ha sido posible, ya que no es una tarea fácil lograr como objetivo la sostenibilidad del sistema económico. Sin embargo, se deben estudiar aquellos puntos en donde convergen el medio ambiente y el sistema económico, de tal forma de contestar la siguiente pregunta: Cómo se puede utilizar el medio ambiente de manera que lo aprovechemos como fuente de mejor calidad de vida, sin llegar a los limites de la irrecuperabilidad, y que sea compatible con la sostenibilidad de un sistema económico?

En este artículo se tratará de explicar, como se podrían contestar algunas de las preguntas anteriormente citadas, en un marco de tipo económico, definiendo en primer lugar, el significado de "Desarrollo Sostenible" y la condición básica para lograrlo que es mantener el stock de capital natural constante; este requisito implica obviamente la existencia de límites, donde el ambiente juegue su rol en el sistema como sostén de la economía. 


\section{El desarrollo sostenible}

En los últimos años el medio ambiente ha pasado a ser un importante motivo de preocupación mundial. En la mayorla de los paises industrializados se considera que la contaminación es una amenaza. Entretanto, el mundo en desarrollo reconoce que la contaminación y la degradación de los recursos naturales se ha tornado un obstáculo para el desarrollo económico y la mitigación de la pobreza. Actualmente, los paises exploran el concepto de desarrollo sostenible, enfoque que permitirá continuar mejorando la calidad de vida con menor consumo de los recursos dejando asi para las generaciones futuras una reserva sin mengua o incluso mayor de recursos naturales $y$ demás patrimonio (CMMAD, 1987; Pezzey 1993).

El concepto de desarrollo sostenible ha evolucionado de forma tal que abarca tres puntos de vista principales: económico, social y ecológico. Los tres conceptos deben conciliarse y ponerse en práctica a fin de alcanzar efectivamente el desarrollo sostenible (Munasinghe, 1993).

El problema se presenta porque se debe de tener en cuenta por un lado los beneficios que ofrece la naturaleza al consumir sus recursos; y por el otro lado, se debe conocer el daño que se ocasiona al medio ambiente. Esto se pone de manifiesto en nuestra dependencia cada vez mayor al sistema de vida que nos han fabricado y que el ambiente nos ha asegurado hasta estos momentos. Es muy cómodo por parte de los agentes contaminantes: individuo, empresa o estado, deshacerse de todo aquello que el mundo moderno, lleno de plástico y tecnologla se ha encargado de surtirnos. Hasta dónde llegar?, esa es la pregunta?. Que clase de productos no debemos consumir?. Tenemos que detenernos y retroceder. Cuales son los sustitutos de los rlos, lagos, océanos, montañas, árboles, animales, etc.?

La condición mínima para que el desarrollo sea sostenible es que el stock de capital natural no disminuya con el tiempo. Considerando que el stock de capital natural es el stock de recursos naturales disponibles sobre la tierra, se podría hablar entonces de dos tipos de stock de capital natural: uno existente, que serla aquel stock existente en el momento de tomar cualquier decisión; y uno optimo que seria aquel stock protector de los recursos naturales (Pearce, 1989).

Existen costos y beneficios derivados de los cambios producidos en el stock de capital natural, si dicho stock disminuye debido a la utilización que le da el hombre a la naturaleza, trayendo un beneficlo como consecuencia de la ganancia obtenida por la utilización del recurso natural, se ve por tanto como el ambiente reduce los costos de producción. Sin embargo, el destruir el ambiente también implica una serie de costos.

Desde el momento que un gran número de personas utilicen un determinado recurso natural, ya sea con un objetivo de tipo espiritual, como por ejemplo al admirar la naturaleza por placer, o con un objetivo de tipo cientffico como por ejemplo para la búsqueda de nuevas medicinas; en la medida que se destruya o se contamine la naturaleza estos beneficios que provienen del uso disminuirán, hasta 
llegar un momento en que se perderían totalmente.

Conceptualmente, el valor económico total (VET) de un recurso consta de su valor de uso (VU) y su valor de no uso (VNU). El valor de uso se puede dividir a su vez en valor de uso directo (VUD), valor de uso indirecto (VUI) y el valor de opción (VO) (valor de uso potencial, es decir el uso que puedo darle en un futuro, tanto como individuo, como agente externo, o como el uso que le darán las generaciones futuras). Una categoría importante del valor de no uso es el valor de existencia (VE) (es decir, el valor intrínseco al objeto, en este caso el ambiente).

Por lo tanto podemos escribir:

$\mathrm{VET}=\mathrm{VU}+\mathrm{VNU}$

$V E T=V U D+V U I+V O$

podemos dejar claro que el ambiente natural posee no solo un valor de uso, sino también un valor de existencia. (Munasinghe, 1994)

La Figura $\mathrm{N}^{2} 1$ representa el confrontamiento entre los costos y los beneficios, en el eje horizontal se indica el stock de capital natural y en el eje vertical se indican los costos y los beneficios.

$\mathrm{Kn}$ : representa el stock optimo de capital natural.

B: Valor económico total : valor de uso + valor de existencia (beneficios).

C: Valor perdido por el desarrollo (costos).

La curva de costos (C) muestra como al incrementar el stock de capital natural (Kn) crecen los costos en forma de beneficios perdidos por no haber conservado el ambiente.

La curva de beneficios (B), representa los beneficios tanto para aquellos que utilizan como para aquellos que no utilizan los recursos naturales.

Figura 1

Costos y beneficios de la conservación ambiental.

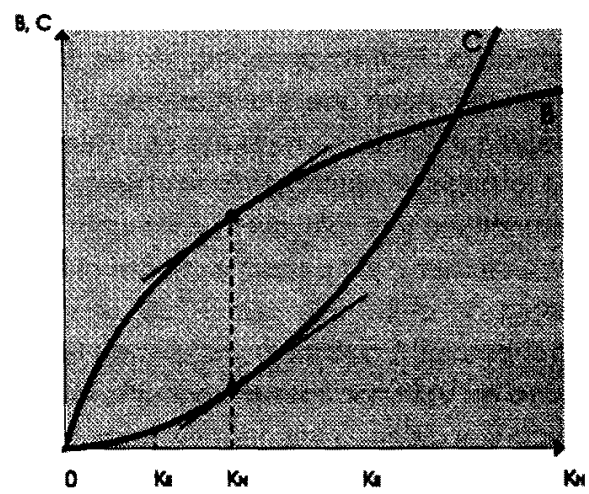

Fuente: Pearce, 1990

La Figura N 2 es resultado de una modificación en la Figura $\mathrm{N}^{2} 1$, se observa un pico, el cual está en correspondencia con el nivel sptimo de $\mathrm{Kn}$, es decir, el stock existente y el stock óptimo de capital natural coinciden, esto en términos de la idea de "capital constante"; por ésta razón la curva de beneficios se transforma en una recta.

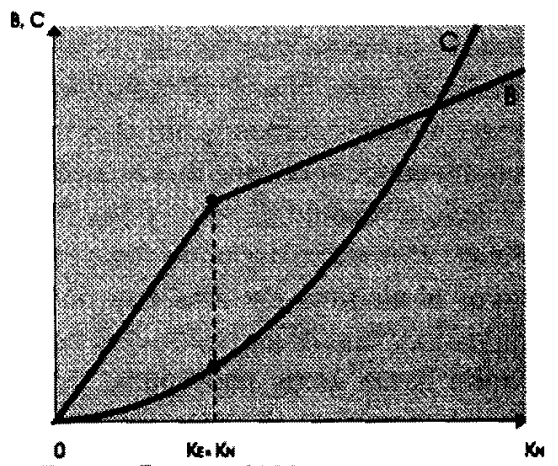

Fuente: Pearce, 1990 
En el contexto de un desarrollo sostenible está implicito que se debería atribuir un valor elevado a la reducción del stock existente de capital. Confirmando que la conservación del stock existente de capital natural debería estar como una de las prioridades básicas (Pearce ot al, 1990).

Para la teorla económica es importante conservar el stock óptimo de capital natural como condición de la sostenibilidad, pero es importante también conservar el stock existente, por ejemplo el caso de los palses en vias de desarrollo que dependen básicamente de los recursos naturales, el stock optimo estará muy por encima del stock existente, y debemos tomar en cuenta factores muy importantes como son la irreversibilidad y la incerleza. Por ejemplo, la explotación de recursos hídricos para riego puede exigir la construcción de un embalse; la aparición de productos alternativos o la caida de los precios podrían hacer que la producción de cultivos regados no sea rentable y ser imposible o muy costoso quitar el embalse y restablecer el curso natural del río. Otro ejemplo se presenta con la conversión de tierras pluviales en agrícolas, a diferencia de los efectos relacionados con la destrucción del capital creado por el hombre, que usualmente puede reconstruirse (excepto el acervo cultural) los proyectos pueden ser causas de la pérdida de capital natural (peces, paisajes, arreciles de coral, etc.) para siempre.

La irreversibilidad no es solo consecuencia de la destrucción de un bien singular (como una zona de bosque pluvial, o un paisaje determinado); también puede producirse al utilizar el medio am- biente de manera no sustentable. Por ejemplo, el fracaso de algunos proyecto de riego en países africanos obedece al aumento de la salinidad (que en casos extremos imposibilita el uso de la tierra). Vale decir que la irreversibilidad también puede ser la consecuencia indeseable de los proyectos de desarrollo (Gómez, 1994).

Si se pudiese tener la certeza de que la sustitución del capital natural por capital producido por el hombre no constituyen un grave problema para la humanidad, sería todo mucho más fácil, pero en realidad las interacciones del medio ambiente con el sistema económico son bastante complicadas y en gran parte inciertas; por tanto la decisión de sustituir el capital natural, destruyéndolo y contaminándolo, trae como consecuencia la irreversibilidad.

Estos dos problemas dan la pauta de que lo más indicado es aumentar el grado de investigación, de tal forma de lograr una más acertada información, para no correr riesgos tan altos que perjudiquen sin fundamento, ni al medio ambiente, ni al nivel de vida, ni a la eficiencia del proceso tecnológico.

Para entender cómo podría interrelacionarse el medio ambiente con el sistema económico, se desarrollarán ciertas reglas, partiendo de un modelo cerrado (sin tomar en cuenta el comercio internacional), y considerando que el ambiente tiene dos funciones principales las cuales son: proveernos de recursos naturales y asimilar los desperdicios.

Las reglas de nuestro juego (reglas de la sostenibilidad) serán las siguientes: 
1) Se debe emplear los recursos renovables de tal forma que lo que se emplee no sea mayor a la capacidad de regeneración natural, tomando en cuenta que el Stock de Capital natural debe ser constante en el tiempo (no consideramos por ahora los recursos no renovables ya que su stock no puede mantenerse constante en el tiempo).

2) Los desperdicios deben ir a la par de la capacidad de asimilación del ambiente.

Si respetan estas dos reglas sabemos que el stock de recursos renovables y el stock de asimilación de los desperdicios, no disminuirán y a futuro será posible seguir sosteniendo el sistema económico. No podemos permitir de ningún modo la disminución ni del stock de recur. sos renovables, ni del stock de asimilación.

Posteriormente al introducir la utilización de recursos no renovables tendremos entonces, que ésta debe compensarse, con un incremento de los recursos renovables, y también debemos tener en cuenta que se debe permitir que un determinado nivel de vida pueda estar garantizado con una determinada reducción del stock de recursos naturales.

Existe otro factor que hasta los momentos no se ha considerado que es el crecimiento demográfico, podemos sostener un cierto nivel de vida con una cantidad menor de recursos en el tiempo, pero al aumentar la población aumenta la demanda de recurso, Qué se debe hacer ahora?

Entonces para, conseguir la sostenibilidad de un sistema económico, se deberla garantizar el desarrollo renun- ciando sólo a una pequeña parte del stock de capital natural, y si deseamos mayor disfrute de los recursos ambientales, el nivel de vida a través del progreso tecnológico no debe aumentar.

En otras palabras el desarrollo sostenible está implícito en una serie de estrategias y políticas que puedan ser utilizadas de tal forma y que incluyan recursos de todo tipo (naturales, humanos, financieros, f(sicos), para lograr aumentar el nivel de vida y el bienestar del ser humano, tomando como condición básica un stock de capital constante, respetando el valor de uso y el valor de existencia del medio ambiente, sin olvidar a las generaciones futuras (Repetto, 1986).

\section{Estrateglas}

\section{Estrategla de cooperación en- tre paises}

Si se analiza el problema del medio ambiente, desde el punto de vista de una economía abierta, donde existe interdependencia estratégica de juego para enfrentar la problemática ambiental global, se observa, que el diagnóstico generalizado ha sido que los patrones de crecimiento mundial no están llevando a un desarrollo sostenible desde el punto de vista del medio ambiente (Varas, 1995). Por tanto se plantean una serie de estrategias a seguir de tal modo de lograr acuerdos entre paises. Estos acuerdos determinarán las acciones a seguir por parte de los distintos palses que forman parte del acuerdo sean estas cooperar conjuntamente considerando el valor actual de la ganancia de una estrategia de 
cooperación o tomar el camino de la deserción.

\section{Estrategla de la minimización de la pérdida máxima}

Otro tipo de estrategia a seguir es el caso de la minimización de la pérdida máxima, si un país esta inseguro en cuanto a la decisión sea esta desarrollar o presenvar el medio ambiente, se plantearán dos hipótesis:

a) Desarrollar o b) Preservar

Cuando el pars se decide por una estrategia de desarrollo la pérdida máxima será igual económicamente al beneficio que se tendría en caso de haber preservado los recursos.

Bd: Beneficio debido al desarrollo.

Bp: Beneficio debido a la preservación.

En caso de $B d>B p$, el país opta por desarrollar solo sí Bd es muy alto, en caso contrario se decide entonces por una estrategia de preservación.

\section{Estrategias y políticas para Amórlca Latina}

El problema de los paises en vías de desarrollo no es adecuarse a una escala de producción de aquellos más ricos, sino de encontrar un equilibrio satisfactorio entre los recursos y los deseos.

Hacemos un alto para explicar que es necesario distinguir entre los deseos absolutos y los deseos relativos. Los de800 absolutos tienen su limite es decir tienen un punto de saciedad. Por ejemplo no necesitamos 10 neveras para ver satisfecha la necesidad de tener nevera, a lo sumo necesitaremos 1 ó 2. Los deseos relativos son insaciables y se podrian identificar en un plano psicológico o social como aquellos deseos de envidia social, de competencia, de imitación y de rivalidad; aquí el límite sería la frustración.

Hay que tomar en cuenta que en cada época la idea de bienestar cambia considerablemente. El problema básico del hombre en nuestros tiempos es hacer frente a todo tipo de deseos, gracias a los cuales han surgido las innovaciones técnicas. El punto está en que ya nada satisface la curiosidad ni el ansia infinita de desear lo no existente; a tal punto, como escribib Ortega y Gasset (1935) en su libro "Meditación de la técnica" el hombre se ha olvidado de que forma parte de la naturaleza.

Si fuésemos más inteligentes no destruiríamos ni contaminariamos nuestro hábitat, sólo por añadir una unidad más de bienestar. Al contrario tuviésemos la capacidad de conservarlo, aprovechándolo de un modo preciso, añadiendo más de una unidad de bienestar, ya sea para el hoy como para el mañana.

Está claro que la evolución técnica nos ha brindado comodidades y beneficios, pero se ha llegado a un punto máximo en donde poco a poco la tecnologia ha ido destruyendo a la naturaleza, al igual que el hombre ha dejado atrás, el ser natural y se ha vuelto un autómata, lleno de deseos insatisfechos, compulsivo e infeliz. Qué quedará para las próximas generaciones?

La pobreza, el hambre, la miseria y la desesperación van de la mano con el grave problema ambiental, producto de la falta de conciencia e inhumanidad de se- 
res humanos autómatas, que al no sulrir las necesidades básicas, se han dedicado a traspasar la frontera de los deseos, por mediación de la tecnología.

En consecuencia, el hombre se aprovecha de las ventajas técnicas para transformar la naturaleza destruyéndola y contaminándola, para lograr satisfacer deseos, tanto relativos como absolutos, con el objeto de incrementar su nivel de vida y sentir satisfacción sin importarle las consecuencias.

La humanidad debería posponer la frontera de sus deseos. Los países en vias de desarrollo se encuentran con el grave problema que encuentran tecnologla diferente a la de los países desarrollados que les permiten aumentar el nivel de crecimiento utilizando mucho menos recursos y mucho más trabajo.

Por lo tanto cada país debe encontrar su propia tecnologla, se necesita inventar tecnologías apropiadas, y que las ayudas internacionales sean en términos no sólo de financiamiento, sino en términos de inteligencia que permita definir la nueva tecnología que logrará aumentar la productividad de los recursos, sin degradar el medio ambiente, y que a su vez no impida, ni ponga en situación de desventaja en términos de competitividad internacional a los paises latinoamericanos.

El verdadero problema tecnológico es tener tecnología de reciclaje no de destrucción; es decir, tecnología que sea capaz de utilizar un mínimo de recursos naturales y energéticos, sin disminuir la productividad ni la competitividad internacional.

Se puede tener una economía estacionaria y un alto nivel tecnológico pa- ralelo a un alto nivel de satisfacciones materiales y espirituales, pero en el caso de los parses con altos indices de pobre$\mathrm{za}$, no se les puede hablar simplemente de sostenibilidad ambiental, ya que para ellos la prioridad básica es la alimentación; y como dice el Prớ. José Lutzem. berger: "las fuerzas del mercado como funcionan hoy, son completamente ciegas a las necesidades de la mayor parte de la humanidad" (Lutzemberger, 1995).

Un ejemplo lo tenemos en la Selva Amazónica, la cual constituye la más grande reserva del mundo de elementos nutritivos y de biotipos, los Rlos Orinoco y Amazonas contienen $1 / 4$ del agua potable disponible en la Tierra (Forstner y Wittmann, 1981), asi como las bases fundamentales para la investigación cientiffca farmacéutica.

De tal forma que el aprovechar los recursos como por ejemplo utilizar la madera para investigación, seguramente aportaría muchos más beneficios tanto desde el punto de vista económico como desde el punto de vista de preservación de la vida en nuestro planeta.

Con todo esto no queremos proponer que se torne a la tecnologia pre-industrial, sino buscar tecnologías adecuadas a una economía cíclica y que envuelva en un todo el proceso cíclico de producción.

En zonas del mundo donde la desocupación es tan alta, necesitamos ocupar un mayor número de personas preservando el recurso natural que tenemos.

Recordando que como países en vias de desarrollo tenemos como tarea fundamental aumentar el nivel de creclmiento. 
El reto consiste en hacer compatibles las polfticas públicas encaminadas a la promoción de un crecimiento económico estable y aquellas dirigidas tanto al control de la contaminación como al aprovechamiento sostenible de los recursos naturales. destacar:

Entre estas polficas se pueden

a.- Las inversiones en recursos humanos a través de programas de educación, salud, nutrición y planificación familiar;

b.- Eliminación de los subsidios a los precios de los recursos energéticos y el suministro de agua porque alientan el uso excesivo de éstos recursos;

c.- El incremento de las inversiones en infraestructura para el suministro de agua potable y saneamiento, que ofrezcan rentabllidad económica, beneficios sociales y ambientales.

d. - Los programas de combate a la pobreza extrema, toda vez que este sector de la población es el que tiene menos medios para protegerse del deterioro ambiental.

e.- De mucha importancia para 10. grar un desarrollo ambiental adecuado es fundamental un sistema legal eficiente y efectivo que permita un desarrollo autónomo sostenible, dentro del mismo sector privado.

f.- En cuanto al rol del Estado: es necesario un Estado no Intervenclonista pero capaz de mantener politicas amblentales de mercado muy claras. Las politicas tendrlan que tender hacia la no intervención y a su vez a fomentar eficiencla y desarrollo amblental (Varas, 1995).

En resumen, los instrumentos para una politica ambiental podrian ser agrupados de la siguiente manera:

- Campañas de concientización y convencimiento.

- Inversión gubernamental.

- Control directo.

- Incentivos económicos: (cobros por contaminar, permisos comerciales, esquemas de depósitos y reembolso, subsidios).

Los dos primeros instrumentos no constituyen en si mismos una forma de integrar los costos ambientales en las decisiones de los agentes económicos. Sin embargo, son instrumentos que deben estar presentes en el diseño de una estrategia integral de protección al medio ambiente.

Los instrumentos de control directo consisten en la aplicación de normas y reglamentos que imponen un limite permisible a las emisiones de contaminantes o bien especifican los procesos y equipos que deberán utilizarse para proteger el medio ambiente. Por ejemplo, la regulación en México está basada casi en su totalidad en el control directo y tiene un alto nivel de detalle (Varas, 1995). En general este tipo de medidas representa ventajas en algunos casos, sin embargo, el uso intensivo de controles directos tiene la desventaja de que requiere evaluar directamente a cada unidad de contaminaclón.

L08 Instrumentos económicos afectan los costos y beneficlos asoclados a un conjunto de medidas que inducen un cambio de conducta en favor de una mayor protección del ambiente. Entre éstos pueden estar:

A.- Impuestos por contaminar: 
Impuestos por descargas de contaminantes en el aire, agua y suelo, y por generación de ruido. Deben estimarse y cobrarse por adelantado y ser devueltos cuando el contaminador demuestre que redujo sus emisiones.

- Impuestos a los usuarios de un sistema público de tratamiento de residuos contaminantes, para cubrir los gastos del mismo, incluyendo gastos de administración y recolección.

- Impuestos a los productos dañinos al medio ambiente, incluyendo materias primas, insumos, bienes de consumo final y desechos.

B.- Esquemas de depósitos y reembolso:

Consisten en el pago de un depósito por la producción y comercialización de productos que representan una amenaza potencial para el medio ambiente. cuando se evitan los efectos contaminantes, mediante el retorno de los productos o de sus residuos, se lleva a cabo un reembolso. C.- Subsidios:

Si bien los subsidios generalmente son una violación al principio "el que contamina paga" ; podrian justificarse en algunas actividades de investigación y desarrollo de tecnologías para reducir la contaminación.

\section{Conclusiones}

En conclusión se puede entender $\theta$ desarrollo sostenible como un proceso de expansión de la economía que puede mantenerse en el tiempo, disfrutando cada generación de una calidad de vida mayor. El motor de este proceso seria la acumulación de capital humano y la pro- ducción de conocimiento y de información pertinente.

El concepto de desarrollo sostenible plantea la idea de crecimiento estacionario per cápita, por lo tanto, cada generación habrla de disfrutar de una calidad mayor en el nivel de vida que su predecesora; se presenta por tanto un concepto de sostenibilidad, configurado por un gran número de elementos, pero sin detallar de la forma en que estos intervienen.

Como recomendación, aparte de las politicas generales que se proponen para que una economla alcance el desarrollo sostenible, se ha llegado a la conclusión de que se debe estimular el tema de la educación ambiental en donde cabe distinguir al menos dos objetivos:

Por una parte, para lograr en la sociedad una adecuada compresión de los desaflos futuros en materia ambiental no solo se debe difundir el conocimiento existente, sino es necesanio enfatizar también los costos de medidas proambientales, subrayando que una decisión en pro del medio ambiente obliga también a sacrificar otros objetivos, por lo tanto, la educación ambiental, debe estimular las preferencias humanas enfatizando la conveniencia de las metas ambientales, el otro objetivo, sería el de desarrollar el conocimiento necesario para que el Indlviduo pueda concebir las consecuencias de sus actos y lo que esto implica para la sociedad.

Otra recomendación que se plantea para los países de América Latina es el desarrollo de tecnologías de reciclaje y la instrumentación de políticas que incentiven económicamente el desarrollo sostenible. 


\section{Referencias Bibliográficas}

CMMAD (Comisión Mundial sobre medio ambiente) (1987). Our Common future, Oxford, Oxford Unlversity Press.

Forstner, $U$ and Wittmann, G. (1981). Metal Pollution In the Aquatic Environment. Springer-Verlag. Berlin. Germany.

Gómez, C. (1994). El anállsis costo-beneflclo y el medlo ambiente. CEPAL, Santiago de Chile. Chile.

Lutzemberger, J. (1995). Revista Brasileira de Administracao. Forum internaclonal de Administración. Miaml, Florlda. U.S.A.

Munasinghe, M. (1994). Aspectos amblentales y decisiones económicas en los paises en desarrollo. CEPAL, Santlago de Chile. Chlle.
Ortega y Gasset, J. (1935). Meditaclón de la tócnica. Madrid

Pearce, D., Barbier, E. and Markandya, A. (1990). Sustainable Development, London Environmental Economics Centre, London. England.

Pearce, D. and Turner, B. (1989). Economlcs of Natural Resources and Environment, London, Hemel Hempstead, Harvester and Wheatsheaf. England.

Pezzey, (1993). Economlc Analysls of sustainable growth and sustalnable development, Worid Bank Environment Paper Nro. 2, Banco Mundial Washington, D.C

Repetto, R. (1986). World Enough and TIme, New Haven, Conn.: Yale University Press. U.S.A.

Varas, J. (1995). Economía del medio amblente. Universidad Católica de Chile, Santiago. Chile. 